\title{
Le télétravail des cadres : entre suractivité \\ et apprentissage de nouvelles temporalités
}

\author{
Jean-Luc Metzger ${ }^{\mathrm{a},}{ }^{,}$, Olivier Cléach ${ }^{\mathrm{b}}$ \\ ${ }^{a}$ Laboratoire Usages, créativité, ergonomie (UCE), France Télécom \\ Recherche \& Développement, 38-40, rue du Général Leclerc, \\ 92794 Issy-les-Moulineaux cedex 9, France \\ ${ }^{b}$ Groupe de recherches en sciences sociales, BP 3337, 27033 Évreux cedex, France
}

\section{Résumé}

Le télétravail est souvent considéré comme un moyen d'améliorer l'articulation entre vie professionnelle et vie privée. Toutefois, pourquoi certains salariés, et singulièrement des cadres, en viennent-ils à choisir cette modalité ? S'agit-il d'ailleurs bien d'un choix ou de l'adoption d'une solution «faute de mieux », dans un contexte organisationnel contraignant? Et dans ce cas, à quels arbitrages temporels procèdent-ils ? Par ailleurs, dès lors qu'ils ont opté pour le télétravail, comment les cadres parviennent-ils à accommoder les contraintes professionnelles et familiales ? Leurs aspirations à une meilleure conciliation sont-elles satisfaites ou rencontrent-ils des difficultés insoupçonnées? Le présent article apporte des réponses à ces différentes questions, en s'appuyant sur deux études menées auprès de cadres pratiquant différentes formes de télétravail. Il souligne, finalement, que l'absence d'une régulation institutionnelle de l'articulation entre vies privée et professionnelle loin d'assurer une existence plus équilibrée, présente des risques sur l'efficacité collective.

(C) 2004 Elsevier SAS. Tous droits réservés.

\section{Abstract}

À traduire en anglais

\footnotetext{
* Auteur correspondant.

Adresse e-mail : jeanluc.metzger@rd.francetelecom.com (J.-L. Metzger).
} 
(C) 2004 Elsevier SAS. Tous droits réservés.

Mots-clés : Télétravail ; Temps de travail ; Cadres ; Nouvelles formes d'organisation du travail ; Temps sociaux ; TIC

Keywords:

\section{Introduction}

Le télétravail donne lieu à de multiples appréciations. Ses promoteurs (des pouvoirs publics comme la Datar et la Commission européenne, des associations de développement du télétravail, certains télétravailleurs indépendants) veulent y voir une modalité pour mieux équilibrer vie professionnelle et vie privée. Ses détracteurs (notamment certains syndicats) y voient, au contraire, un instrument de flexibilité, un risque de destruction des identités professionnelles et une menace pour la capacité d'action collective. S'il a parfois été envisagé comme moyen d'aménagement du territoire, de revitalisation rurale, il est surtout révélateur de l'évolution des firmes dans le cadre de la globalisation ${ }^{1}$. Selon la définition retenue, l'observateur pourra conclure à son caractère marginal ou, au contraire, à sa récente extension (Ordioni, 2000). Certains y verront l'archétype du travail dominé féminin (parce qu'à domicile et faiblement qualifié), alors que les études les plus récentes montrent au contraire qu'il concerne principalement les cadres supérieurs (très majoritairement masculins) (Emerit, 2000). Ces quelques réflexions soulignent plusieurs axes d'investigation.

Tout d'abord, elles rappellent l'importance, non seulement, de clarifier le sens donné à ce terme, mais également de préciser qui est à l'initiative de l'introduction du télétravail (la direction de l'entreprise, le management local ou le salarié) et dans quelle mesure cette modalité de travail est choisie par ceux qui s'y engagent (fussent des cadres). De plus, apparaît la nécessité d'identifier en quoi sa pratique réelle modifie l'articulation entre les différents temps sociaux.

Pour rendre compte de ces différentes facettes, nous allons présenter les résultats de deux études (une qualitative et une quantitative) menées auprès de cadres

\footnotetext{
1 Pour une étude comparative du télétravail au niveau européen, voir (Emerit, 2001). Sur l'articulation entre télétravail et mondialisation économique, voir (Beer, 2001).
} 
pratiquant différentes modalités de télétravail (Cléach et Metzger, 2002 ; Cléach et Metzger, 2003). Mais, auparavant, qu'entendons-nous exactement par télétravail ?

\section{Le télétravail : une définition délicate et contingente}

Étymologiquement, ce terme signifie « travail à distance ». Mais par rapport à quoi le travail est-il distant? Par rapport au client, au donneur d'ordre, au public, au produit final ? Et quel statut possède le télétravailleur ? Comment ses prestations sont-elles rémunérées, évaluées ? Comment sa production est-elle livrée ?

En réalité, face à cette gamme étendue de situations distantes possibles, et en nous limitant au seul cas des salariés, deux types de télétravailleurs peuvent être distingués: ceux exerçant des emplois administratifs d'exécution, pratiquant le télétravail à domicile à plein temps, le plus souvent des femmes qui peuvent se retrouver professionnellement isolées ; et ceux qui occupent des emplois de cadres supérieurs, pratiquant un télétravail occasionnel et inscrits dans des collectifs professionnels. On peut y voir deux idéaux-types opposés du télétravail, cas limites des tendances entre lesquelles fluctuent, sans jamais s'y réduire, les évolutions du travail. Leur correspondent deux définitions ${ }^{2}$.

La première, que l'on peut qualifier de restrictive, voit dans le télétravail une modalité d'exercice de l'activité inscrite dans le contrat de travail, selon laquelle l'intégralité du temps de travail (temps complet ou partiel) se déroule au domicile du salarié, la mise en relation avec l'entreprise (ou le donneur d'ordre) étant le plus souvent assurée au moyen des technologies de l'information et de la communication.

La seconde, que l'on peut considérer comme extensive, celle adoptée par un nombre croissant d'observateurs et par la Commission européenne ${ }^{3}$, inclut d'autres modalités. Tout d'abord, la pratique du télétravail peut être occasionnelle et ne pas se traduire par une modification du contrat de travail (télétravail informel). Ce qui conduit à tenir compte du télétravail alterné (alternance régulière de jours travaillés à domicile et de jours travaillés dans l'entreprise) et du télétravail en débordement (le

\footnotetext{
${ }^{2}$ Les définitions et typologies de télétravail sont discutées de façon plus exhaustive dans (Bérard, 2002 ; Largier, 2001 ; Lemesle et Marot, 1994).

Comme l'indique l'accord cadre européen sur le télétravail, du 16 juillet 2002.
} 
salarié poursuit ses activités professionnelles, le soir ou le week-end, voire pendant les vacances, en retrouvant son environnement de travail). Le lieu d'exercice peut être le domicile, l'hôtel, les transports en commun (mobilité). Ces situations se distinguent du simple fait d'apporter des dossiers à la maison. En effet, par le biais de la mobilisation systématique des outils de communication et de traitement de l'information (applications, bases de données, outils «collaboratifs »), le travail de bureau, et donc les relations d'échanges à distance avec les collègues se prolongent pendant le temps libre : le télétravailleur en débordement est pour ainsi dire vingtquatre heures sur vingt-quatre connecté à ses préoccupations professionnelles et interagit avec les différentes sources de sollicitation (mails, forums, appels téléphoniques sur le portable, etc.).

Nous retiendrons cette seconde définition pour des raisons empiriques et théoriques. Empiriquement, elle correspond, non seulement aux situations les plus fréquentes rencontrées dans nos observations, mais également à celles caractérisant des pratiques émergentes chez les salariés européens. En effet, des recherches récentes, résumant plusieurs études comparatives, montrent que «le télétravail alterné, le télétravail mobile et le télétravail occasionnel supplantent aujourd'hui très largement les formes anciennes de télétravail», étant entendu que «la grande majorité des télétravailleurs d'aujourd'hui ont des tâches qualifiées et un niveau de formation élevé » (Emerit, 2001). Natacha Ordioni précise que l'on trouve « $59 \%$ de diplômés de l'enseignement supérieur parmi les télétravailleurs, contre $27 \%$ dans l'ensemble de la population » (Ordioni, 2000). Ce que confirme indirectement Alain Chenu en notant que les cadres et ingénieurs font fréquemment déborder leur vie professionnelle sur leur temps familial et domestique en effectuant certaines de leurs tâches à domicile (Chenu, 2002).

Par ailleurs, une telle approche du télétravail nous paraît pertinente également d'un point de vue théorique, car elle donne à voir une dimension souvent occultée du travail des cadres et, plus généralement, de la flexibilisation dont les activités qualifiées sont l'objet. De plus, elle permet de questionner le degré d'autonomie dont disposent précisément ces salariés : dans quelle mesure s'engagent-ils librement dans ces modalités de télétravail ? Certes, en apparence, cette flexibilité paraît négociée, contractuelle (Ordioni, 2000) et donc, jusqu'à un certain point, consensuelle. Mais une analyse du «monde vécu » des télétravailleurs cadres, notamment de la manière 
dont ces derniers articulent sphère professionnelle et sphère familiale, nous permettra de relativiser cet a priori.

Pour conclure, nous retiendrons que le télétravailleur est un salarié qui, de façon relativement régulière et pendant une période variable, exerce ses activités professionnelles en dehors de son entreprise habituelle de rattachement, maintenant un contact avec celle-ci et ses collègues par l'usage de procédés de télécommunications (téléphone, internet/courriel, fax...). Durant une partie de ses activités, le salarié qui travaille à distance ne se trouve plus en contact physique avec son équipe de travail ni sous la supervision directe de son supérieur hiérarchique, celui-ci ne pouvant procéder qu'à un contrôle a posteriori (Cléach et Metzger, 2003).

\section{Population étudiée}

L'étude quantitative par questionnaire à laquelle nous avons procédé au sein d'une entreprise de services (278 réponses exploitées) montre que les télétravailleurs sont très majoritairement des cadres supérieurs (66 \% alors qu'ils ne sont que 13,5\% dans l'entreprise) et des hommes (77\%, contre $63 \%$ dans l'entreprise). «Notre» télétravailleur type est un homme, âgé de 43 ans, marié, père de famille, diplômé, cadre supérieur ayant 17 années d'ancienneté, gros utilisateur de TIC, exerçant ses activités d'experts, plus que de management. Cette distinction est importante: comme le montre notre étude qualitative, l'activité de management, de commandement, demeure liée à la proximité physique du responsable et $\mathrm{du}$ subordonné. Les managers pratiquant le télétravail se heurtent à une réticence de leurs subordonnés et de leurs supérieurs (Cléach et Metzger, 2002). Par ailleurs, notre télétravailleur exerce son activité dans les domaines «commercial-vente » ou «des techniques informatiques » et, depuis 1 à 2 ans, travaille à distance en débordement au domicile $(97 \%)$ et, occasionnellement, de façon nomade (81\%) ou alternée $(51 \%)^{4}$. Il consacre entre 15 et $20 \%$ de son temps de travail à cette modalité, soit tout de même près d'une longue journée de cadre par semaine. Cet engagement dans

\footnotetext{
${ }^{4}$ On retrouve cette surreprésentation masculine et de cadres supérieurs parmi les 33 salariés effectuant du télétravail contractualisé alterné deux jours par semaine.
} 
le télétravail a souvent été précédé par l'habitude d'apporter du travail à domicile (45\%), voire pendant les vacances $(30 \%)$.

L'étude qualitative a porté sur une population de 25 cadres travaillant dans la même entreprise de services. La plupart sont des experts techniques (télécommunication, informatique) et fonctionnels (gestion du personnel, juriste, marketing), souvent (mais pas toujours) férus d'informatique, de bureautique ou de multimédia. Ils se sont engagés de longue date dans le télétravail qu'ils pratiquent (parfois de façon «militante »), selon la répartition suivante : 7 sont essentiellement des travailleurs alternés ; 8 télétravaillent surtout en débordement ; 10 sont avant tout « nomades ». Enfin, $80 \%$ des télétravailleurs de notre échantillon avaient le statut « de cadre exécutif autonome ».

\section{Transformation du temps de travail et volonté de télétravailler}

Pour comprendre l'attrait que peut présenter le télétravail pour les cadres, il faut rappeler que cette modalité s'inscrit dans les transformations de l'organisation $d u$ temps de travail, engagées grâce à la mobilisation des dispositifs techniques et tout particulièrement les $\mathrm{TIC}^{5}$. Dit autrement, le télétravail, en tant que dispositif de gestion ${ }^{6}$, constitue l'un des instruments de la flexibilisation et de l'injonction à agir dans l'urgence qui ont cours dans certaines entreprises. Il apparaît comme un indicateur d'analyse pertinent d'évolution des temps sociaux. À ce titre, rappelons certains éclairages fournis par la sociologie du temps.

\subsection{Temps et technique : les apports de la sociologie}

Charles Gadéa et Michel Lallement (Gadéa et Lallement, 2000) rappellent qu'Émile Durkheim (Durkheim, 1979) a, le premier, montré que le temps (tant du point de vue de l'idée que l'on s'en fait, que des pratiques qu'il sous-tend) n'était pas une catégorie a priori de l'entendement (comme le soutenait Emmanuel Kant), mais résultait d'une organisation collective. Dans la voie ainsi ouverte, les études du temps

\footnotetext{
${ }^{5}$ Sur l'articulation entre TIC et travail, voir (Craipeau, 2001 ; Muhlmann, 2001).
} 
se sont d'abord centré sur l'observation des pratiques professionnelles : l'analyse du temps de travail industriel a longtemps servi de "paradigme» aux analyses des temps sociaux, y compris celles du « hors-travail ».

D'ailleurs, William Grossin souligne que l'utilisation des innovations technologiques accompagnant les décisions d'organisation, «en modifiant profondément le rapport du travail au temps et à sa rémunération », contribue à structurer, non seulement le temps de travail, mais également les représentations sociales du temps (y compris les catégories d'analyse mobilisées par les chercheurs) (Grossin, 1998).

Sur cet arrière-fond, comment définir les frontières du temps de travail ? M. Lallement distingue temps au travail (celui résultant de l'organisation du travail), temps $d u$ travail (celui permettant, via l'emploi et la formation, d'acquérir un statut social) et temps de travail (le temps qui s'oppose au hors-travail) (Lallement, 2003). Ce qui nous permet de préciser que le temps de travail est celui consacré à l'activité professionnelle et inclut non seulement le temps de transport, et celui de la mobilisation subjective, mais également le temps de formation, consistant, de plus en plus, à s'approprier le fonctionnement de procédures et de dispositifs techniques, en dehors de toute inscription à un cours ${ }^{7}$.

Il est d'autant plus important d'avoir à l'esprit ces distinctions, que la pratique du télétravail rend plus indéfinies les frontières entre travail et hors-travail. Et ce, de deux manières : d'une part, comme modalité d'aménagement du temps de travail, dans un contexte où les utilisations systématiques des TIC «sont empreintes d'une flexibilité qui se répercute sur les modalités temporelles de l'organisation du temps de travail » (Grossin, 1998); d'autre part, en ce que pratiqué à domicile, il empiète sur la sphère domestique.

\subsection{Organisation du temps de travail et TIC}

En effet, sans emboîter le pas des discours annonçant que l'introduction des TIC conduit nécessairement à l'émergence de «l'entreprise en réseau » et

\footnotetext{
${ }^{6}$ Pour une présentation du télétravail comme dispositif de gestion, voir (Metzger et Cléach, 2004).

${ }^{7}$ Ces définitions précisent les réflexions de Georges Friedmann pour qui le travail ne s'oppose pas mécaniquement aux activités menées pendant les loisirs (Friedmann, 1964).
} 
« révolutionne » l'organisation du travail ${ }^{8}$, on peut retenir que la mobilisation de ces dispositifs dans le cadre professionnel interagit de plusieurs manières avec le temps de travail, sa durée (son contenant) et son organisation (son contenu ${ }^{9}$ ). Les TIC, en tant qu'éléments d'une stratégie managériale, peuvent être des instruments de l'intensité temporelle du travail (voir 4.2.1., ci-dessous). Elles peuvent engendrer sans que personne ne l'ait expressément cherché — une surcharge de travail (4.2.2.). Elles sont, enfin, des marchandises dont certains salariés (les supports produits, les « marketteurs », les commerciaux) doivent chercher à développer les usages, ce qui les amène à consacrer un temps considérable à cette activité (4.2.3.). Examinons ces différentes voies.

4.2.1. En matière d'initiatives managériales, Y. Queinnec, B. Barthe et F. Verdier rappellent qu'il existe six manières d'accroître l'intensité temporelle du travail salarié, parmi lesquelles l'intensification (les cadences croissent plus vite que la durée du travail ne diminue) et la densification ou chasse aux temps morts (Queinnec et al., 2000).

On voit mieux ici combien, par des choix organisationnels appropriés, l'introduction des TIC peut être mise au service de l'intensité temporelle. Qu'il s'agisse de la messagerie électronique, des outils de travail collaboratif, du téléphone portable (Jauréguiberry, 2003) ou de gestion des processus, leur inscription dans l'activité professionnelle permet d'imposer la productivité par l'augmentation des cadences et par la lutte contre les temps morts. C'est le cas dans les centres d'appels (Lechat et Delaunay, 2003). mais c'est également ce que connaissent régulièrement les cadres experts de l'entreprise étudiée (informaticiens, ingénieurs réseau,

${ }^{8}$ À propos de ces représentations « emphatiques sur la nouvelle informatique », voir les critiques bien venues de David Muhlmann (Muhlmann, 2001). Quant à l'identification de moments-charnières (les « révolutions techniques »), elle « est révélatrice de la tendance des hommes à faire table rase du passé toutes les fois qu'ils estiment avoir opéré une transmutation des valeurs » (Gurvitch, 1958, p. 322).

${ }^{9}$ W. Grossin distingue deux dimensions du temps de travail : le contenant, c'est-à-dire les aspects externes, quantitatifs, comme la durée formelle ; et le contenu, c'est-à-dire les normes, les rythmes, la cadence, les pauses (Grossin, 2000). Ce qui permet de tenir compte, pour un même contenant (la même durée globale), d'une grande diversité de temps, selon les types de contenus. C'est, par exemple, sur le contenu que se cristallise la «chasse aux temps morts », l'une des modalités de la rationalisation du temps — au sens de Max Weber, voir (Lallement, 2003, pp. 58-59). Quant aux salariés soumis à de longues heures de travail, comme les cadres, l'essentiel est pour eux, à contenant inchangé, d'assouplir les horaires ou de réaménager le temps de travail lui-même (voir Pronovost, 1998 ; Bouffartigue et Bocchino, 1998 ; Bouffartigue et Boutillier, 2000 ; Chenu, 2002). 
concepteurs de services, etc.). En effet, les postes d'assistant et de soutien logistique étant supprimés, les activités correspondantes leur sont tout bonnement transférées (seuls les cadres dirigeants conservant un secrétariat).

«En tant que cadre, on est quand même pas mal sollicité, on a des horaires de travail qui sont extensibles. Il faut que je fasse des journées qui commencent à $8 \mathrm{~h}$ et qui se terminent à $20 \mathrm{~h}$. Ça va un moment, mais on ne peut pas faire ça tout le temps, sinon ça fait des semaines d'une longueur horrible. » (André L., expert informatique, télétravail en débordement et alterné occasionnel.)

La responsabilisation qui accompagne la réduction du nombre de niveaux hiérarchiques, s'appuie souvent sur la mise à disposition d'applications informatiques permettant de suivre la productivité et de la comparer à celle des «pairs », ce qui renforce la pression temporelle ${ }^{10}$.

Par ailleurs, le fait de pouvoir se connecter à distance et à tout moment, au réseau interne de l'entreprise (donc, de pouvoir utiliser les applications informatiques, les bases de données, vingt-quatre heures sur vingt-quatre), renforce, certes, la possibilité de contrôler l'assiduité des travailleurs nomades, mais surtout la pression diffuse (et jamais explicitée) pour faire disparaître les temps de pause et élargir sans limite précise la plage de temps pendant laquelle il est souhaitable de travailler. C'est ce que nous indiquons à la section suivante.

4.2.2. En matière d'effets non prévus, l'usage des TIC présente une double réalité à laquelle les aspirants au télétravail n'échappent pas :

- d'une part, la facilité offerte dans le travail au quotidien (dossiers partagés, capacité à joindre aisément les personnes par e-mail sans avoir l'impression de les déranger, etc.) rend plus confortables certaines activités liées aux échanges d'informations ;

- et d'autre part, l'utilisation intensive (et parfois hors de propos) du courrier électronique exacerbe certaines contraintes professionnelles (surcharge d'informations ou de données, engendrant une surcharge cognitive, mais aussi

10 Pour une description détaillée du caractère incessant des transformations technicoorganisationnelles touchant notamment les cadres de cette entreprise, voir (Metzger, 2000). 
se traduisant par un allongement de la durée du travail nécessaire pour traiter tous les messages et n'avoir pas le sentiment de se «noyer ») ${ }^{11}$. L'un de nos interlocuteurs nous signalait ainsi qu'il recevait 90 mails par jour, soit près de 1000 en deux semaines (quantité qu'il doit traiter à son retour de congés).

Il faut également tenir compte du fait que les dispositifs informatiques sont sans cesse l'objet de mise à jour ou de remplacements (versions) qui conduisent l'utilisateur d'un ensemble donné d'outils à se perfectionner sans cesse, à se tenir au courant, à maîtriser les différentes interfaces et compatibilités entre logiciels et matériels, ce qui, là encore, constitue des activités gourmandes en temps ${ }^{12}$.

4.2.3. Sous la pression réelle des entreprises concurrentes, mais aussi sous la pression d'une compétition qui s'apparente à une sorte de jeu entre pairs, il n'est pas rare que, dans l'entreprise étudiée, des responsables du développement des ventes (d'un produit ou d'un service nouveau) se sentent obligés de multiplier les initiatives pour trouver de nouveaux marchés. Ce qui les conduit à présenter des idées de service à des professionnels en dehors des heures ouvrables (salons, réunions ad hoc, expérimentations). Ils se sentent également obligés d'utiliser eux-mêmes ces marchandises, là encore en dehors des heures ouvrables, pour mieux en connaître les possibilités et les limites, afin d'en promouvoir la vente avec plus de pertinence.

Enfin, certains cadres commerciaux cherchent à être tenus au courant des initiatives de leurs pairs et de leurs concurrents, en pratiquant une veille commerciale incessante, y compris la nuit et pendant les vacances, en particulier par la consultation des différentes sources d'informations disponibles sur internet. Cette vigilance de tous les instants prend parfois la forme d'une compétition ludique entre cadres supérieurs, se lançant des défis à $23 \mathrm{~h}$, pour être le premier à dénicher une information.

\subsection{L'engagement dans le télétravail : un choix largement contraint}

${ }^{11}$ Sur les usages professionnels de la messagerie et ses impacts temporels, voir par exemple (Saintive, 2000). L'auteur parle de densification temporelle.

${ }^{12}$ Pour Heinrich Schwarz, Bonnie A. Nardi et Steve Whittaker les efforts pour maitriser, sélectionner, mettre à jour, rendre compatibles, voire acheter les dispositifs techniques requis pour travailler à distance font partie de ce qu'ils appellent le coût caché du travail virtuel, évidemment à la 
Dans ce contexte, les évolutions de l'organisation du travail et la mobilisation des TIC, en allongeant substantiellement le temps consacré à l'activité professionnelle, orientent la décision des cadres de s'engager dans le télétravail. En effet, nos observations montrent que les raisons de l'engagement dans les différentes modalités de télétravail sont essentiellement professionnelles :

- près de $48 \%$ des répondants à l'enquête par questionnaire disent pratiquer le télétravail, d'abord, pour terminer des dossiers tout en étant à domicile ;

- $23 \%$ pour des raisons liées à la nature de leur activité (pouvoir consulter et traiter les mails dans les meilleurs délais ; afin de mener à bien des projets ou de suivre, même en congé, l'évolution d'un projet sensible ; pour répondre à une surcharge de travail ; à cause de nombreux déplacements en région ou à l'étranger ; à cause de l'existence d'astreintes hors temps ouvrable);

- près de $15 \%$ pour des raisons liées à la situation géographique de leurs interlocuteurs (parce que l'équipe projet ou l'équipe de collaborateurs est répartie sur tout le territoire ou sur plusieurs sites ; du fait du décalage horaire avec des filiales implantées à l'étranger);

- $2 \%$ suite à un déménagement du service.

C'est également un moyen d'améliorer l'organisation du temps de travail, de manière à être plus productif, en particulier en évitant de «perdre du temps » à être dérangé au bureau. Ce qui revient, en fait, à supprimer les pauses ${ }^{13}$. Pour d'autres télétravailleurs, c'est une façon d'atténuer les conséquences de l'allongement de la plage de temps consacrée au travail, plage au sein de laquelle se glissent des intervalles « improductifs » qui consistent souvent à attendre (attendre une réponse à un message, attendre la parution d'une information dont le salarié, sous contrainte d'objectifs et de délais, a besoin pour sa réflexion).

Il faut tenir également compte du fait que l'ARTT, en réduisant le nombre de jours travaillés à effectifs constants, accroît la charge et les rythmes de travail quotidiens, plaçant les cadres autonomes devant un dilemme : rester plus tard au bureau ou achever à domicile une partie de leurs tâches. Le second terme de l'alternative peut

seule charge du (télé)travailleur (Schwarz et al., 1999).

${ }^{13}$ On retrouve ici l'analyse de Laurent Taskin (Taskin, 2002) ainsi que celle Matthieu de Nanteuil (Nanteuil, 2002). 
alors être préféré, comme un compromis vis-à-vis de la famille (voir les enfants avant qu'ils ne soient couchés) et une compensation en termes de confort de travail.

Enfin, rien ne nous laisse supposer qu'il se produise un effet d'imitation (voire un sentiment de jalousie) ou d'entraînement entre salariés, permettant d'expliquer un processus de diffusion/d'extension du télétravail. Même au sein des équipes de cadres constituées, il n'existe pas de pression systématique pour que tous pratiquent le télétravail (notamment nomade et en débordement, et encore moins alterné). Par ailleurs, la légitimité du télétravail n'est pas suffisante pour que cette forme soit enviée.

Si les répondants au questionnaire n'ont pas signalé de raisons personnelles pour justifier le choix de télétravailler, les entretiens qualitatifs montrent que l'engagement dans le « télétravail » apparait aussi comme une manière de réduire la part consacrée au temps de travail (qui inclut le temps de trajet).

«Pourquoi le télétravail ? Pour ne plus être dans des conflits terribles que j'ai vécus pendant des années et des années. [...] Les sommes de conflits de priorités dans lesquels on est mis, c'est dur. [...] J'étais obligée de courir, de regarder ma montre le soir pour aller, par exemple, chez le pédiatre ou pour un rendez-vous chez mon médecin. Je le prenais à $19 \mathrm{~h} 30$. Je regardais ma montre. Je m'impatientais dans les embouteillages. J'étais crevée le soir.» (Isabelle F., activités fonctionnelles, télétravail alterné un jour par semaine.)

Certains cadres peuvent alors changer de fonction (passant de l'opérationnel au fonctionnel) pour pouvoir télétravailler, le meilleur équilibre résultant alors $\mathrm{du}$ changement de fonction et de la pratique du télétravail. Celle-ci n'est donc que très modérément un choix, mais plutôt une manière de rendre compatible quatre injonctions: réaliser un nombre croissant de tâches complexes pour une durée donnée ; s'adapter à une organisation de plus en plus éclatée et changeante ; répondre dans des délais très brefs aux demandes de renseignement, en partie la conséquence de l'utilisation des messageries électroniques (culte de l'urgence) ; et enfin, ne pas passer un temps jugé exorbitant au bureau.

Dit autrement, à première vue, l'engagement dans le télétravail semble relever d'une logique propre aux salariés, le management se contentant de laisser faire. Mais 
une réflexion plus poussée montre que c'est bien l'accumulation des décisions managériales qui, en façonnant l'environnement du travail, conduit les cadres experts, déjà enclins à ne pas compter leur temps, à trouver désirable de télétravailler.

À côté de ces «bonnes raisons», il ne faut pas oublier que l'extension du télétravail nécessite également un certain nombre de conditions préalables, telles que l'acquisition d'équipements techniques adéquats (notamment l'ADSL) dont le coût est supporté le plus souvent par le salarié ${ }^{14}$, ainsi que l'édification d'une pièce dédiée au domicile ${ }^{15}$. Enfin, la pratique du télétravail requiert des arrangements familiaux (ou un certain cloisonnement des différents moments domestiques et professionnels) : le salarié ne peut télétravailler à certains moments privilégiés de la journée, comme le repas pris en famille, le couchage des enfants, voire le suivi des devoirs scolaires ; symétriquement, le conjoint et les enfants ne peuvent déranger le télétravailleur pendant son activité professionnelle à domicile. Comme nous allons l'analyser plus bas, ces derniers prérequis ne vont pas de soi et nécessitent la construction de compromis, un apprentissage culturel.

\section{La pratique du télétravail : des résultats ambigus}

Toutefois, cette option aboutit-elle au résultat espéré ? La pratique des différentes formes de télétravail permet-elle une meilleure efficacité professionnelle et simultanément, de mieux se réaliser dans ses activités familiales et de loisirs? En réalité, l'analyse montre que la frontière entre les sphères privée et professionnelle étant brouillée par la pratique du télétravail, l'articulation entre les temps sociaux doit être repensée.

\subsection{Le risque d'aggraver les contraintes temporelles}

Concrètement, une journée de télétravail est «saucissonnée » en tranches: professionnelles à domicile, familiales à domicile et professionnelles au bureau ou en

\footnotetext{
${ }^{14}$ L'entreprise ne prend en charge tout ou partie des frais d'équipement et de consommation téléphonique que dans le cas du télétravail contractualisé.

${ }^{15}$ Obligatoire dans le cas du télétravail contractualisé.
} 
déplacement. Mais le fait de savoir qu'il n'y a pas d'heure pour recevoir (et donc traiter) un mel toujours susceptible d'être urgent (au moins dans les imaginaires), conduit le télétravailleur à rester connecté sur des plages de temps très étendues ${ }^{16}$.

Ici les TIC ne sont pas seules en cause : la posture même de télétravailleur, laissé libre d'organiser son temps, sans garde-fou formel et impératif, peut non seulement induire des dépassements d'horaires, mais également remettre en cause le délicat équilibre des temps sociaux. L'extension du temps de travail peut alors réellement être produite par la pratique du télétravail, au lieu d'être réduite ${ }^{17}$.

«C'est quelque chose dont on ne se rend pas compte, mais [...] devant cette liberté de pouvoir prendre un dossier ou autre, c'est de se faire piéger à travailler après le boulot. Et ça, c'est extrêmement dangereux, parce qu'au bout d'un moment, il y a une sorte d'état de fatigue qui s'instaure, parce qu'on ne fait pas la coupure [...] et on peut tomber au boulot, même si c'est chez soi, ce qui est paradoxal. » (Jacques N., chef de département, télétravail en débordement.)

La préservation de l'équilibre entre les différents temps sociaux ne va pas de soi : sous la pression d'une sorte de «culpabilité » intériorisée (culpabilité de pouvoir travailler dans de bonnes conditions, en échappant à certaines contraintes superflues comme le temps de transport, le bruit engendré par la vie de bureau, etc.), le travailleur à distance est tenté d'en faire trop, d'en rajouter, en un mot, de travailler sans limite ${ }^{18}$. Pour résister, il faut avoir appris à se fixer des objectifs, mais également à s'imposer des bornes. Savoir «quand arrêter le travail» devient une compétence à part entière.

En cela, il y a réellement nécessité de disposer, préalablement à la pratique du télétravail, de capacités d'autonomie ${ }^{19}$. Mais, simultanément, la pratique du

${ }^{16}$ Ce qui est facilité par le fait que l'ADSL libère la ligne téléphonique.

${ }^{17}$ Notre étude quantitative montre que, pour les cadres pratiquant le télétravail en débordement, l'empiètement sur le hors travail représente 10 à $20 \%$ de la durée du temps de travail total.

${ }^{18}$ La question de la culpabilité est développée dans (Cléach et Metzger, 2002). Plus généralement, le fait d'être livré à soi-même pour organiser son temps, conduit le télétravailleur à travailler plus longtemps (Taskin, 2002).

${ }^{19}$ Ici, la notion d'autonomie : d'une part, renvoie à l'aptitude à définir son identité professionnelle et construire sa capacité d'action (voir, par exemple, Liu, 1981); d'autre part, elle souligne le fait que, parce que les règles définissant le travail sont nécessairement incomplètes, les salariés doivent 
télétravail peut renforcer l'apprentissage de cette capacité, faute de quoi, non seulement la croissance du surtravail conduit à dégrader la qualité du travail, mais l'empiétement sur la vie privée est vivement critiqué par les proches (tel ce cadre supérieur dont les enfants sont obligés de cacher, pendant les vacances, le microordinateur portable pour l'amener à prendre conscience de leur existence). On peut alors soutenir que, dans les situations favorables (en particulier, un environnement familial réclamant la présence du télétravailleur), la pratique du télétravail à domicile, par l'apprentissage qu'elle permet, rend l'intéressé apte à assumer la quête d'une meilleure articulation des temps sociaux.

Plus généralement, la promesse d'auto-organisation des différents temps sociaux rencontre des limites, à l'instar des autres mesures d'aménagement du temps de travail qui, «loin de résoudre les problèmes de compatibilité temporelle, [...] peuvent en faire surgir de nouveaux » (Gadéa et Lallement, 2000). Cette limitation résulte de la difficulté à construire la capacité (individuelle, mais aussi collective) à organiser l'articulation entre les différents temps (et les différentes sphères), difficulté résultant des choix organisationnels privilégiant la compétition interindividuelle et les réorganisations permanentes.

Mais au-delà de la seule question des temps, le travail à distance n'agit-il pas également sur les pratiques privées (domestiques, familiales), le contenu des activités hors travail $^{20}$ ? En réalité, cette influence est réciproque et s'exprime de deux manières. D’une part, les télétravailleurs (particulièrement les alternés contractualisés) semblent mieux profiter de leur vie hors travail. Mais d'autre part, la pratique du télétravail présente le risque de brouiller les frontières entre espaces privé et professionnel.

\subsection{Articulation entre pratiques professionnelles et espaces privés}

Georges Gurvitch, dans les années 1950, avait souligné l'importance de la multiplicité des temps sociaux («un des plus importants que la sociologie ait

forcément les interpréter, les compléter (Reynaud, 1989 ; Terssac, 1992). Enfin, sur la question de l'autonomie en rapport avec le télétravail, voir (Kouloumdjian, 1999).

${ }^{20}$ Comme nous l'avons indiqué, ce type de questionnement déborde le seul télétravail et fait partie des fondements de la sociologie du travail (Friedmann, 1964 ; Dumazedier, 1964). Plus récemment, Bernard Lahire aborde également cette question (Lahire, 2002). 
rencontré sur son chemin »(Gurvitch, 1968, tome 2, p. 327). Il notait que la constitution de groupes ou d'institutions s'accompagne - pour se coordonner, évoluer, décider - de la mise au point de temporalités spécifiques qui manifestent une grande permanence. Dès lors, chaque individu étant inscrit dans plusieurs groupes et institutions (par exemple, le travail et la famille), « sa vie est soumise au calendrier de chacun d'eux ; ils viennent interférer dans sa personne » (p. 326). Mais toute tentative individuelle pour faire concorder les différentes configurations temporelles se heurte aux efforts collectifs d'harmonisation. C'est précisément le type de difficulté que rencontrent les télétravailleurs chez qui la nécessité de gérer l'articulation des temps sociaux prend un relief accru.

Sachant également que, comme le souligne Monique Haicault, l'utilisation des TIC, en introduisant des temporalités presque instantanées, accroît le caractère contradictoire et hétérogène des temps sociaux, les activités non professionnelles continuant à s'inscrire dans la durée (Haicault, 1998) ${ }^{21}$. Les salariés sont alors contraints de jongler avec ces différentes temporalités, au point qu'ils doivent prendre le temps de planifier, d'organiser la manière dont ils vont faire s'entremêler au mieux leurs différentes activités, rémunérées, domestiques, familiales, ludiques. L'auteur parle de «capacités virtuoses de gestion complexe des temps et des pluritemporalités sociales », capacités que les individus doivent acquérir. Ce point est particulièrement structurant dans le cas du télétravail.

En optant pour cette forme de travail (alterné et en débordement) comme ressource, les salariés privilégient une certaine réactivité par rapport à leurs proches (garder un enfant malade, par exemple) ou pour le moins, une présence rassurante au domicile. Même s'ils n'effectuent aucune activité avec leur famille (puisqu'ils travaillent au domicile), leur simple présence constitue en soi une modification substantielle et appréciée (notamment le mercredi où les enfants peuvent rester à la maison, sans déranger celui des deux parents qui télétravaille). Des activités nouvelles peuvent être pratiquées, comme s'occuper des devoirs scolaires des enfants (tel ce père qui tient à rentrer suffisamment tôt pour donner des leçons de

\footnotetext{
${ }^{21}$ Notons que les TIC contribuent à brouiller les frontières entre travail et hors-travail par un autre biais. En effet, de nombreux individus commencent par utiliser des logiciels ou des services de téléinformatique, dans un cadre domestique. Ce faisant, ils apprennent à maîtriser toutes sortes de savoirs et savoir-faire qu'ils peuvent ensuite réutiliser dans leur activité professionnelle. Cette pratique n'est pas passée inaperçue aux yeux des managers qui encouragent l'usage domestique des TIC en
} 
mathématiques à sa fille). Mais, le plus souvent, c'est la manière de réaliser les activités domestiques qui évolue, qui gagne en qualité, du simple fait de pouvoir les organiser sans précipitation. Le télétravail (le plus souvent alterné) permet, dans certaines situations, d'ajuster au mieux la production professionnelle et les soins familiaux (attention pour soi et les proches) et de s'en acquitter l'esprit plus tranquille, plus apaisé :

«Le fait de pouvoir bosser chez moi le vendredi [...] cela me permet d'avoir un rendez-vous, par exemple chez le médecin à $10 \mathrm{~h}$ du matin, quitte à démarrer à 7 h 30 dans mon travail. [...] Ça permet de rendre moins incompatibles des activités qui sont quand même assez incompatibles. » (André L., expert informatique, télétravail en débordement et alterné occasionnel.)

De même, une majorité de répondants (54\%) se disent «dé-stressées », particulièrement vis-à-vis des trajets domicile/travail, de l'obligation de terminer un dossier le vendredi (le dossier peut être terminé le samedi matin, en deux heures, tranquillement) et d'être suffisamment à l'écoute des proches (même si, parfois, le micro-ordinateur branché en permanence dans un coin du salon ou dans la piècebureau ou encore apporté en vacances peut jouer le rôle d'un intrus et d'un concurrent).

Néanmoins, la référence à ce confort accru peut relever d'un discours de justification, vis-à-vis d'une activité professionnelle devenue aussi source d'insatisfaction. En réalité, pour que la pratique du télétravail conduise à une meilleure maîtrise des temporalités, les cadres doivent d'abord veiller à ce que le temps libre ne soit pas trop « contaminé» par les exigences professionnelles ${ }^{22}$. Plus précisément, il convient de distinguer deux dimensions : l'accroissement du temps consacré à l'activité professionnelle, et l'accroissement de l'insatisfaction que cela provoque.

En effet, depuis la fin des années 1980, on assiste, non à une extension générale du temps de loisirs, mais à un déplacement de la charge de travail vers les catégories

offrant des ordinateurs, des abonnements, ou des tarifs préférentiels à leurs personnels.

${ }^{22}$ C'est en cela que le temps du télétravail ne se confond pas avec du temps gagné sur le travail (comme celui qui résulte de la réduction du temps de travail). C'est du temps pour mieux travailler. 
les plus qualifiées (Chenu et Herpin, 2002). Mais simultanément, cette évolution s'accompagne d'une augmentation de l'insatisfaction chez les cadres. Là où Maurice Thévenet parle de sentiment d'iniquité et de dissonance forte (Thévenet, 2001), Vanessa Pinto note que les cadres éprouvent «des besoins que le déficit de temps libre, causé par le travail, empêche de combler » (Pinto, 2003). Dans le même sens, Michel Gollac parle de la frustration qu'éprouvent les cadres dans leur vie hors travail : leur famille ou leur entourage leur reprochant de trop se consacrer à l'activité professionnelle (Gollac, 2004).

Cela souligne combien les perceptions du « temps qu'il est normal de consacrer au travail » dépendent, certes, du temps réellement consacré à l'activité rémunérée, mais également du degré de satisfaction/insatisfaction par rapport au(x) temps disponible(s) pour la famille, les loisirs, les autres activités ${ }^{23}$. D'ailleurs, M. Thévenet rappelle que, s'il est parfois pertinent d'opposer travail et hors-travail, ce n'est pas parce qu'il s'agit de deux réalités disjointes, mais parce que chaque individu peut être amené à éprouver des difficultés à articuler ces deux aspects de sa propre existence (Thévenet, 2001). La recherche d'accomplissement de soi dépend de la place relative que chacun accorde au travail et au hors-travail dans la construction de son identité.

Pour en revenir aux télétravailleurs observés, la possibilité de contenir l'extension du temps de travail à domicile n'est pas un exercice aisé. C'est que ces salariés envisagent d'abord le travail et le hors-travail comme des compartiments naturellement étanches, entre lesquels il n'est pas nécessaire de prévoir des dispositifs d'isolation. Dans ces conditions, c'est-à-dire sans précaution, le télétravailleur prend le risque d'accroître la confusion entre les sphères d'activités.

«J'avais 15 jours de vacances, je ne pouvais absolument pas m'en empêcher, c'était comme une drogue, d'aller voir mes mails pour gagner du temps parce que je savais que sinon... j'allais me retrouver avec 1000 mails ou autres, à mon retour de congés. Donc, je préférais les faire d'avance un petit peu. Comme ça, en plus, s'il y avait un feu sur quelque chose, ça me permettait de régler le problème. Mais avec un téléphone portable, l'engrenage y était. C'est-à-dire qu'on devait

${ }^{23}$ Comme le rappelle par exemple Dominique Méda, le travail salarié est loin d'être la seule activité d'une existence sociale complète (Méda, 2000). Pour Joffre Dumazedier, avoir plus de temps libre peut participer à la construction de l'identité professionnelle (Dumazedier, 1988). 
aller à la plage à $9 \mathrm{~h}$ et à $11 \mathrm{~h}$, je venais de finir de régler le problème et j'étais très satisfait, j'étais très content d'avoir réglé mes problèmes de boulot à la plage. Je ne sais pas si maintenant, avec le recul, je le referais. » (Jacques N., chef de département, télétravail en débordement.)

Ce n'est qu'en prenant conscience des effets négatifs de cet entremêlement (dégradation de la qualité du travail et des relations familiales), que le télétravailleur peut développer des compétences dans la maîtrise des temporalités, et ainsi envisager un meilleur équilibre travail/hors-travail. L'apprentissage de cette aptitude à maîtriser les différentes temporalités s'appuie donc sur des expériences parfois malheureuses. Plus généralement, ce sont les salariés pratiquant le télétravail alterné contractualisé, qui parviennent le mieux à l'équilibre travail/hors-travail. Ils développent pour cela des stratégies d'endiguement et définissent un certain nombre de garde-fous. Mais dans cet effort, la situation de télétravail étant caractérisée par une absence de régulation formelle, ils ne peuvent compter que sur eux-mêmes et leurs proches.

Enfin, sur la question de l'articulation pratiques professionnelles et espace privé, il est pertinent de prendre en compte les rapports sociaux de sexe ${ }^{24}$. Ceux-ci peuvent être envisagés sur deux plans. Tout d'abord, en ce qui concerne les télétravailleurs eux-mêmes, notre étude ne permet pas de distinguer significativement la manière dont les télétravailleurs et les télétravailleuses gèrent cette articulation : les salariés des deux genres s'accordent pour dire que le télétravail permet une meilleure conciliation vie privée vie professionnelle, par un temps à domicile plus important (moins de temps passé dans les déplacements), permettant une meilleure participation aux moments de la vie familiale (parfois pour apporter une réponse aux urgences) et, plus généralement, une utilisation plus qualitative du temps. Par ailleurs, on pourrait s'intéresser à la manière dont la pratique du télétravail influe sur la répartition des tâches domestiques entre hommes et femmes. Mais notre étude n'étant pas centrée sur cette problématique, nous ne disposons pas de données empiriques représentatives. Par contre, nous avons pu établir que les proches jouaient un rôle

\footnotetext{
${ }^{24}$ Sur la question de la répartition des tâches domestiques, voir, par exemple (Barrère-Maurisson 2001). Et sur le cas particulier des rapports de genre dans la pratique de télétravail, voir (D'Amours, 2001 ; Tremblay, 2003).
} 
important pour limiter l'extension du temps de travail à domicile et ainsi préserver l'équilibre travail/hors-travail. C'est ce que nous allons voir au point suivant.

\subsection{Aménagement de l'espace domestique et coupures symboliques}

La plupart des personnes interviewées disposent, au domicile, d'une pièce dédiée, réservée au télétravail. Elle peut être aménagée « tout informatique » (reconstitution de l'espace professionnel) ou comme un bureau plus classique, une pièce où l'on aime bien lire, écrire, se retirer pour apprécier le calme.

Globalement, le fait de disposer d'une pièce dédiée est important à plus d'un titre :

- cela permet évidemment de s'isoler et de se concentrer sur des dossiers complexes qui réclament un traitement au calme ;

- c'est aussi une manière de mettre le travail à distance pour les proches, notamment les conjoints qui n'ont pas nécessairement envie d'être confrontés, chez eux, à l'émergence de la sphère professionnelle ;

- cela permet, enfin, de maintenir une frontière entre la sphère professionnelle et la sphère domestique (moyen symbolique de rupture). Sortir de cette pièce, c'est quitter le travail.

C'est alors à un véritable jeu de l'esprit que les télétravailleurs doivent se livrer pour reconstituer le franchissement de la frontière entre deux univers imbriqués. D'autres télétravailleurs mettent au point des techniques d'autocontrainte, soit pour empêcher le «hors-travail » de les distraire de leur tâche, soit pour contenir le travail dans des limites jugées raisonnables.

«Il m'arrive, de temps en temps, deux ou trois fois par mois, de passer une journée, une journée c'est plus rare, de travailler chez moi. Je dis à ma femme : « OK, demain matin je vais rester » ou « demain après-midi, je vais être là ». Je lui dis : «donc, là je travaille ». [...] Ma femme me dit simplement « tu viens manger à telle heure ». Donc, très bien, je note : elle me fixe un rendez-vous à telle heure, je sors pour aller manger!»(Gilles R., expert financier, débordement et parfois alterné.) 
Ce qui nous amène à souligner combien les proches sont partie prenante dans l'articulation des sphères privée et professionnelle. D'un côté, ils participent à la confusion des genres, en sollicitant le télétravailleur pendant les heures de travail, comme ce conjoint qui profiterait bien de la présence de son épouse (télétravaillant) pour se rendre à une réunion. Mais, d'autre part, les proches contribuent à la limitation de cette confusion en rappelant les devoirs familiaux, comme ces enfants qui s'impatientent après $18 \mathrm{~h}$ et le font savoir. Tout se passe dès lors comme si la charge d'encadrer le travail était partagée par le télétravailleur et sa famille : cette dernière constitue même le principal garde-fou contre le surtravail, son rôle consistant à définir la frontière entre travail et hors-travail, activité qui fait partie de ce que H. Schwarz, B.A. Nardi et S. Whittaker appellent le coût caché du travail virtuel (Schwartz et al., 1999).

Ainsi, sans que cela ne soit jamais explicité ni anticipé (au risque donc d'échapper à toute réflexion rigoureuse), le contrôle social dévolu à l'entreprise, est transféré vers l'environnement familial, activité pour laquelle les intéressés ne sont ni légitimes, ni reconnus et encore moins rémunérés ${ }^{25}$. Ce qui souligne l'existence d'un vide dans la régulation entre sphères professionnelle et privée, vide que les entreprises suscitent en déportant sur les individus la responsabilité de la mettre au point, après l'avoir rendue invisible ${ }^{26}$.

\section{Le télétravail : indicateur des évolutions à l'œuvre dans les organisations}

Ainsi, analyser des dimensions apparemment marginales, pour ainsi dire interstitielles, se révèle riche d'enseignements sur le travail ${ }^{27}$. À travers l'engagement des cadres dans le télétravail et la pratique de cette modalité se donne, en effet, à voir le projet de l'entreprise éclatée (ou en réseau), idéalement faite d'individus ayant contractualisé avec un « donneur d'ordre » et où la forme de la charge de la contrainte

\footnotetext{
${ }^{25}$ On ne peut s'empêcher de souligner le parallèle avec l'hospitalisation à domicile qui transfère sur les proches une partie des tâches de soin, jusqu'alors prises en charge par les professionnels de la santé.

${ }^{26}$ D’autres coûts cachés peuvent être recensés : la régulation entre membres d'un collectif de travail, régulation qui s'effectue assez aisément en présentiel, mais qui pose problème à distance.

${ }^{27}$ Voir, à ce propos, l'analyse limpide qu'en fait L. Taskin (Taskin, 2002).
} 
est transférée sur des salariés pour qui l'autonomie octroyée constitue à la fois une opportunité d'enrichissement et un risque d'épuisement. Au moment où certains annoncent l'irrésistible déploiement de l'internet, notamment à des fins professionnelles (Castells, 2001), on voit l'intérêt que présente cette analyse soulignant le caractère ambivalent de l'usage des TIC : à la fois cause de la recherche d'une meilleure efficacité productive, motif de l'engagement dans le télétravail, source potentielle de surtravail facilitant la contamination de la sphère privée et parfois porteuse d'un meilleur équilibre entre les temps sociaux.

Elle montre également combien il est délicat de raisonner en termes de choix ou de volontariat : si de nombreux individus en viennent à télétravailler, c'est, certes, à cause des changements cumulés des rôles sociaux et d'aspirations identitaires, mais surtout de l'organisation du travail dans le sens de sa flexibilisation. La nécessité de réaliser un équilibre travail/hors-travail n'est pas la préoccupation première de l'engagement dans le télétravail, celui-ci fait suite à plusieurs contingences technicoorganisationnelles. Ce n'est qu'une fois installé dans la pratique du télétravail que cette recherche d'équilibre devient saillante.

Finalement, une part importante des télétravailleurs parvient, avec l'appui de leurs proches, à développer des stratégies d'endiguement leur permettant de limiter la confusion des genres entre sphères privée et publique. Mais si ces savoir-faire, pourtant si utiles, ne dépassent pas le cadre de leur propre expérience, au risque de provoquer des déboires chez nombre de leurs collègues tentés par le télétravail, cela provient, en partie, de ce même contexte socio-organisationnel, fait de bouleversements incessants, d'individualisation et de mise en concurrence, réduisant les capacités d'apprentissage collectif (Metzger, 2002). Ainsi, subissant la désinstitutionnalisation des entreprises, même les cadres rencontrent des difficultés d'intégration sociale, du fait que «l'enchevêtrement contradictoire des temps de l'individu et des organisations rend problématique cette injonction propre à la modernité qui impose à l'individu de devenir maître et possesseur de son destin et de sa sociabilité » (Lallement, 2004).

\section{Références}


Barrère-Maurisson, M.A. (Ed.), 2001. Partage des temps et des tâches dans les ménages. La Documentation française, Paris.

Beer, A. (de), 2001. L'essor du télétravail en Europe. Futuribles 266 (juillet-août), $61-65$.

Bérard, D. (Ed.), 2002. Le télétravail en question. Analyse critique à partir de 10 cas d'entreprises. Études et documents de l'Anact, Lyon, février.

Bouffartigue, P., Bocchino, M., 1998. Travailler sans compter son temps ? Les cadres et le temps de travail. Travail et emploi 74.

Bouffartigue, P., Boutillier, J., 2000. Réduire le temps sans réduire la charge ? Les cadres et les 35 heures. Travail et emploi 82.

Castells, M., 2001. La galaxie Internet. Fayard, Paris.

Chenu, A., 2002. Les horaires et l'organisation du temps de travail. Économie et statistique $352-353,151-167$.

Chenu, A., Herpin, N., 2002. Une pause dans la marche vers la civilisation des loisirs ? économie et statistique 352-353, 15-37.

Cléach, O., Metzger, J.L., 2002. Rapport d'étude sur le télétravail en ADSL. France Télécom Recherche et Développement, ${ }^{\circ}$ RP/FTR\&D/7793, Issy-les-Moulineaux.

Craipeau, S., 2001. L'entreprise commutante. Lavoisier, Paris.

Cléach, O., Metzger, J.L., 2003. Le télétravail : une légitimité en construction ? Bilan d'une étude sociologique auprès des salariés d'une grande entreprise de services. France Télécom Recherche et Développement, $\mathrm{n}^{\circ} \mathrm{RP} / \mathrm{FTR} \& \mathrm{D} / 8344$, Issy-lesMoulineaux.

D’Amours, L. (Ed.), 2001. Télétravail. Concilier performance et qualité de vie. Isabelle Quentin éditeur, Québec.

Dumazedier, J., 1964. Travail et loisir. In: Friedmann, G., Naville, P., Traité de sociologie du travail. Armand Colin, Paris, tome 2, pp. 341-366.

Dumazedier, J., 1988. Révolution culturelle du temps libre (1968-1988). Méridiens Klincksieck, Paris.

Durkheim, E., 1979 Les formes élémentaires de la vie religieuse. Le système totémique en Australie. Puf, Paris ( $1^{\text {re }}$ édition en 1912).

Emerit, 2001. Les métamorphoses du télétravail. La lettre Emerit 27.

Friedmann, G., 1964. Le travail en miettes. Gallimard, Paris. 
Gadéa, C., Lallement, M., 2000. Les rationalisations du temps. In: Terssac, G. (de), Tremblay, D.G., 2000. Où va le temps de travail ? Octarès, Toulouse.

Gollac, M., 2004. Le travail pour le meilleur et pour le pire. Informations sociales $114,84-95$.

Grossin, W., 1998. Les temps de travail. In: Coster, M. (de), Pichault, F. (Eds.), Traité de sociologie du travail. De Boeck, Bruxelles/Paris, pp. 139-155.

Grossin, W., 2000. L'apport des recherches sur les temps de travail et les modes de vie. In: Terssac, G. (de), Tremblay, D.G., 2000. Où va le temps de travail ? Octarès, Toulouse.

Gurvitch, G., 1968. La vocation actuelle de la sociologie. Puf, Paris, 2 tomes.

Haicault, M., 1998. Travail à distance et/ou travail à domicile : le télétravail. Nouvelles formes d'emploi, nouveaux contenus de travail des logiques contradictoires. Laboratoire d'économie et de sociologie du travail (Lest), Aix-enProvence.

Jauréguiberry, F., 2003. La mise en place des télécommunications mobiles au sein des entreprises comme vecteur de nouvelles formes d'exploitation. Communication aux IX ${ }^{\text {es }}$ journées de Sociologie du travail, novembre.

Kouloumdjian, M.F., 1999. Télétravail : (En)jeux de rôles pour acteurs multiples. In: Bernaud, J.L., Lemoine, C. (Eds.), Le psychosociologue dans l'entreprise. L'Harmattan, Paris.

Lahire, B. (Ed.), 2002. Portraits sociologiques. Nathan, Paris.

Lallement, M., 2003. Temps, travail et modes de vie. Puf, Paris.

Lallement, M., 2004. Changement des temps et temps des changements. In: Collectif Griot, Figures du temps. Les nouvelles temporalités du travail et de la formation. L'Harmattan, Paris.

Largier, A., 2001. Le télétravail. Trois projets pour un même objet. Réseaux 106, 203-229.

Lechat, N., Delaunay, J.C., 2003. Les centres d'appels : un secteur en clair-obscur. L'Harmattan, Paris.

Lemesle, R.M., Marot, J.C., 1994. Le télétravail. Puf, Paris.

Liu, M., 1981.Technologie, organisation du travail et comportements des salariés. Revue française de sociologie 22 (2), 205-221. 
Méda, D., 2000. Travail et temps sociaux : pour une nouvelle articulation. In: Terssac, G. (de), Tremblay, D.G., Où va le temps de travail ? Octarès, Toulouse.

Metzger, J.L., 2000. La réforme permanente d'un service public : entre utopie et résignation. L'Harmattan, Paris.

Metzger, J.L., 2002. Apports et limites des théories de l'apprentissage organisationnel : le cas de la réforme permanente. Formation emploi 77 (janviermars).

Metzger, J.L., Cléach, O., 2004. Les dispositifs de gestion : entre efficacité, légitimité et construction de collectifs. L'exemple du télé-travail et du télé-enseignement. Communication au $1^{\text {er }}$ congrès de l'AFS, 24-27 février.

Muhlmann, D., 2001. Des nouvelles technologies à l'image des vieilles organisations. Sociologie du travail 43 (3), 327-347.

Nanteuil, M. (de), 2002. Vers de nouvelles formes de vulnérabilité sociale? Réflexions sur les rapports entre flexibilité et précarité. Travail et emploi 89, 65-80. Ordioni, N., 2000. L'accroissement du télétravail en Europe : quelques hypothèses interprétatives. Mondes en développement 28-111, 69-76.

Pinto, V., 2003. Les attitudes des cadres face à la réduction du temps de travail. Centre d'études de l'emploi Quatre pages 55 (janvier), 1-4.

Pronovost, G., 1998. Loisir et travail. In: Coster, M. (de), Pichault, F. (Eds.), Traité de sociologie du travail. De Boeck, Bruxelles/Paris, pp. 95-114.

Queinnec, Y., Barthe, B., Verdier, F., 2000. Réduction du temps de travail et organisation de l'activité de travail : des rapports ambigus et complexes. In: Terssac, G. (de), Tremblay, D.G., Où va le temps de travail ? Octarès, Toulouse.

Reynaud, J.D., 1989. Les règles du jeu. Armand Colin, Paris.

Saintive, B., 2000. Trois formes différenciées d'usage de la messagerie électronique au sein d'une organisation. Réseaux 104, 119-136.

Schwarz, H., Nardi, B.A, Whittaker, S., 1999. The Hidden Work in Virtual Work. International Conference on Critical Management Studies, Manchester, 14-16 July.

Taskin, L., 2002. Télétravail : enjeux et perspectives dans les organisations. Humanisme et entreprise 303 (février), 81-101.

Terssac, G. (de), 1992. L'autonomie dans le travail. Puf, Paris

Thévenet, M., 2001. Vie professionnelle, vie privée et développement personnel. Revue française de gestion (juin-août), 106-119. 
Tremblay, D.G., 2003. Le télétravail : quels impacts sur l'organisation du travail ? Communication aux $\mathrm{IX}^{\mathrm{es}}$ journées de Sociologie du travail, novembre. 